\title{
Electrical conductivity of cement mortar with the addition of graphene
}

\author{
Tatiana Potses ${ }^{1}$, Vladimir Novikov ${ }^{2}$, Kirill Sergeev $^{2}$, and Sergey Leonovich ${ }^{1,3}$ \\ ${ }^{1}$ Department building materials and construction technology, Belarusian National Technical \\ University, Minsk (Belarus) \\ ${ }^{2}$ Laboratory of Physical and Chemical Technologies. of the National Research Center of the National \\ Academy of Sciences of Belarus for Materials Science \\ ${ }^{3}$ Qingdao University of Technology, 266033, China, 11 Fushun Rd, Qingdao
}

\begin{abstract}
With the development of technological innovations in the field of construction, increasing the efficiency of energy-saving materials used, more and more attention is paid to electrically conductive materials. Electrical conductivity studies were carried out on samples of concrete with graphene addition to obtain the current-voltage characteristics of the material. In order to identify the mechanism of the effects of different concentrations of graphene on electrical conductivity, a series of samples with different concentrations of graphene was made. As a result of the research, it was found that the dependence: the resistivity of the materialthe concentration is percolation, the concentration of graphene in the amount of $8 \%$ of the mass of cement is the percolation threshold.
\end{abstract}

\section{Introduction}

The scope of application of electrically conductive heating elements made of concrete is quite extensive, it can be as the creation of anti-icing structures of roads, sidewalks, reducing the humidity of underground structures, heating concrete at a young age during construction at a sub-zero temperature, it is important to note that giving electrically conductive properties to structural concrete should be while maintaining the initial strength characteristics of conventional concrete.

In various studies, graphite, fullerene, carbon nanotubes, carbon fiber, steel microwires and other conductive additives were introduced into concrete. Figure 1 shows the schematic arrangement of conductive inclusions in concrete with different elongation.
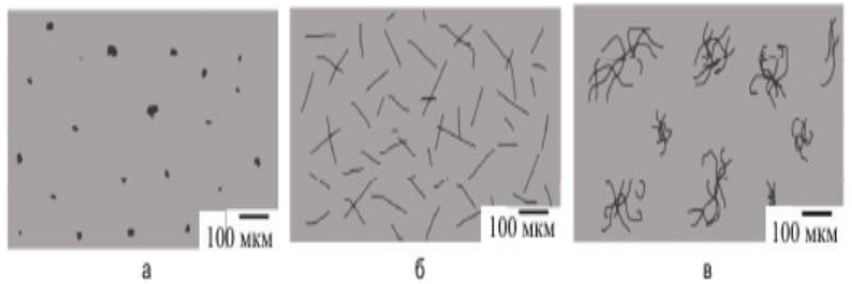

Fig.1. Schematic representation of the concrete structure with various conductive inclusions: concrete with graphite particles (a); concrete with short fibers (b); concrete with long fibers (tangles) [1].

Concrete with introduced graphite microparticles is known, it has a low electrical resistance, but its significant drawback is the need to introduce large (up to $30 \mathrm{vol} \%$ ) graphite concentrations. Indeed, graphite has a spherical shape, and in order to create 
conductivity through the entire sample, it is necessary to ensure that graphite particles contact each other, and for round particles this is feasible only at their high concentrations. At a graphite concentration of $30 \%$, the strength of concrete is reduced by 10-30 times, which is unacceptable for most applications, so such concrete has not found application. From the point of view of ensuring conductivity, it is advisable to use conductive particles with a large elongation - rods, fibers, therefore, in a number of works, nanotubes, carbon fiber and other objects with a large elongation were introduced into concrete. The results with carbon nanotubes can be considered successful, since the conductivity was achieved with their insignificant content [1].

The production of domestic nanomaterials, in particular graphene, on the basis of the Scientific and Practical Center of the NAS of Belarus for Materials Science, makes it possible to improve technologies related to various composite materials. The joint work of the Research Laboratory of Industrial and Civil Engineering of BNTU and the Scientific and Practical Center of the NAS of Belarus for Materials Science involves the development of electrically conductive concrete, which is a composite material with the addition of graphene (5-8\%), having electrical conductivity with a resistivity of the material, which is then proposed to be used as an electrically conductive structural and heating material. The influence of various carbon nanomaterials on the electrical conductivity of cement composites was determined in studies [2].

It is well-known that the electrical resistivity of cement-based composites varies with age due to changes of the amount of pore water $[3,4]$. The electrical resistivity of the composites including conductive nanomaterials also changes as the curing age increases if the amount of nanomaterials included is below the percolation threshold. This is because the pore water helps to establish a continuous conductive pathway for current flow [5]. For instance, as the pore is filled with water, a continuous conductive pathway is established, as shown in Figure 2a, whereas if the water in the pore evaporates, the conductive path becomes disconnected, as shown in Figure $2 b$.

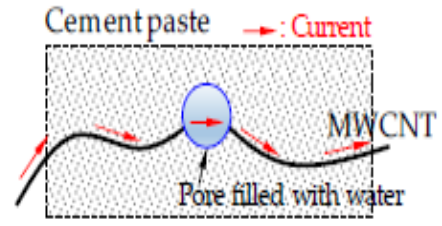

(a)

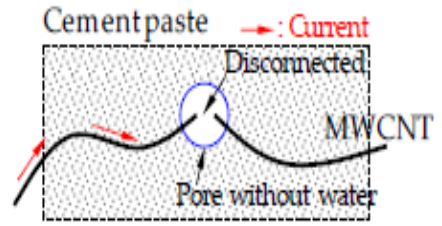

(b)

(a) full saturation condition, (b) dry condition

Fig.2 Schematic description for conductive pathway[2].

Therefore, the electrical resistivity of plain cement paste and cement composites with an insufficient amount of carbon-based nanomaterials varies with the curing age [2].

When cement mortar is hardened, several phases can be distinguished: an aqueous solution of cement, gel formation, crystallization. The formation of the gel is accompanied by hydration of oxides; this process is determined by the mobility of $\mathrm{OH}-\mathrm{Ca}+$ ions, which can be investigated, in particular, by determining the electrical conductivity. Hardening cement can be attributed to molecular crystals, the specific electrical conductivity of which is determined by the ratio [1-3]:

$$
\sigma=\sum \mu i \times \eta i(1)
$$

where $i$ is the type of charge carrier; $\mu i$, $\eta \grave{i}$ is the concentration and mobility of carriers, respectively. In aqueous solution and gel, the charge carriers are ions (in aqueous solution, these ions can be considered free charge carriers) $[6,7]$.

Cement binder is quite a suitable type, since its electrical resistivity is 6-8 orders of magnitude higher than that of carbon, and the coefficient of linear expansion is close to the 
coefficient of linear expansion of the additive. The requirements for the range of permissible operating temperatures are determined by the temperature resistance of cement stone $\left(+150^{\circ} \mathrm{C}\right)$, since carbon allows heating up to $550^{\circ} \mathrm{C}$ [8]. The heating rate depends on the amount of carbon black. The higher its percentage, the faster the material heats up. A composition with $13 \%$ carbon heats up to $50^{\circ} \mathrm{C}$ in 20 minutes, however, taking into account the strength characteristics, a composition with a content of $10-11 \%$ carbon should be adopted [9].

The working hypothesis of the study is that the difference in the coefficient of linear expansion of graphene and cement binder made it possible to assume that the resistivity of electrically conductive concrete will decrease as the concrete strength increases, at the same time, when the percolation threshold is reached, the resistivity will not increase.

\section{Methods}

Prism samples from cement mortar for determining the range of graphene concentrations to identify the percolation threshold.In the first stage of the research, samples with a concentration of graphene by weight of cement of $4 \%, 6 \%, 10 \%$. Two electrodes were installed in the samples, measurements were carried out using a two-probe method.

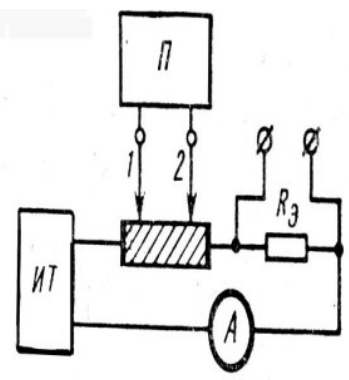

ИТ- current source; A - ammeter;

$\Pi$ - potentiometer; $\mathrm{R}$ et. - reference resistance

Fig.3. The scheme of measuring the resistivity by the two-probe method

According to the measurement results, resistance was observed in samples with a concentration of $6 \%$ and $10 \% 15$ and 31 times lower compared to samples with a $4 \%$ concentration, and 29 and 56 times lower compared to the control samples.

The next stage of the study of the effect of different concentrations of graphene on electrical conductivity was the study of samples of cement-sand mortar with an additive concentration by weight of cement in $5 \%, 6 \%, 8 \%$ by the four-probe method.

Most methods of measuring the specific electrical conductivity of materials are based on determining the voltage drop at some part of the sample through which an electric current is passed. A number of physical effects and phenomena may occur at the contact of the material and the metal electrode during the flow of an electric current, which may introduce a significant error in the measurement results and in some cases make such measurements impossible. The most important for concrete are galvanic effects. These phenomena must be taken into account not only when measuring the specific conductivity, but also in all other cases when an electric current flows through the measured sample with metallic contacts, it is necessary to strive for such experimental conditions that the effect of the phenomena is minimal.

The simplest method for determining resistivity is to measure the resistance of a sample of a regular geometric shape with a constant cross-section. However, this method is not 
suitable for technological purposes due to the complexity of sample preparation and the inability to control the properties of the material in any part of the sample. In addition, the resistivity determined by this method is the average value over the entire volume of the sample and is true only for a homogeneous sample. Probe methods are most often used to measure resistivity, which allow avoiding these disadvantages. In the production and laboratory practice of monitoring the physical parameters of materials, methods of measuring resistivity are widely used, both at direct and alternating current.

A four-probe resistivity measurement method was used for the research, since it is the most common. The main advantage of the four-probe method is that it does not require the creation of ohmic contacts to the sample and it is possible to measure the resistivity of samples of a wide variety of shapes and sizes. The condition of its applicability from the point of view of the sample shape is the presence of a flat surface, the linear dimensions of which exceed the linear dimensions of the probe system. The essence of the four-probe method of measuring resistivity is as follows. Four metal probes are placed along one line on the flat surface of the sample (Fig.4). Current I is passed through two external probes (1 and 4 ), and the potential difference $U$ is measured between two internal probes ( 2 and 3 ). The measured values of the potential difference between probes 2 and 3 , the current flowing through probes 1 and 4, can determine the resistivity of the sample.

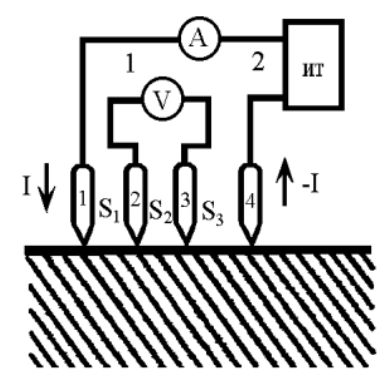

Fig.4 The measurement scheme by the four-probe method.

\section{Test program}

The measurements were carried out on a device manufactured by the Russian company "Electro Chemical Instruments" single-channel potentiostat-galvanostat P-40X. Resistance determination was carried out in the potentiostat mode in the linear scan mode. In the linear scan mode, the device scans the potential or current at a given speed. Measurements of the of the samples were carried out in current-voltage characteristics the ranges from 0 to $5 \mathrm{~V}$ and up to $3 \mathrm{~A}$.

Table 1. Mixture proportions

\begin{tabular}{|l|l|l|l|l|}
\hline No. & Modifier & W/C & $\begin{array}{l}\text { Dosage graphene, \% by } \\
\text { weight of Binder }\end{array}$ & Storage \\
\hline 1.1 & $\begin{array}{l}\text { referent (without } \\
\text { graphene) }\end{array}$ & 0.5 & & $\begin{array}{l}\text { Storage } \\
\text { at } \\
\text { humidity } \\
55 \% \pm 5\end{array}$ \\
\cline { 1 - 3 } 2.1 & \multirow{2}{*}{ Graphene } & 0.5 & 5 & 6 \\
\cline { 3 - 4 } 2.2 & 0.5 & 8 & \\
\cline { 3 - 4 } & & 0.5 & 8 & \\
\hline
\end{tabular}

Plates representing a stainless steel mesh pressed with graphite paper were used as electrodes. 


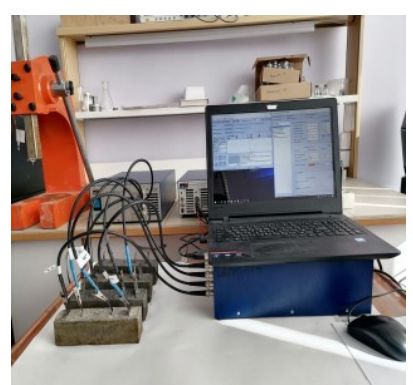

Fig.5.The process of measuring the resistance of samples by the 4-probe method.
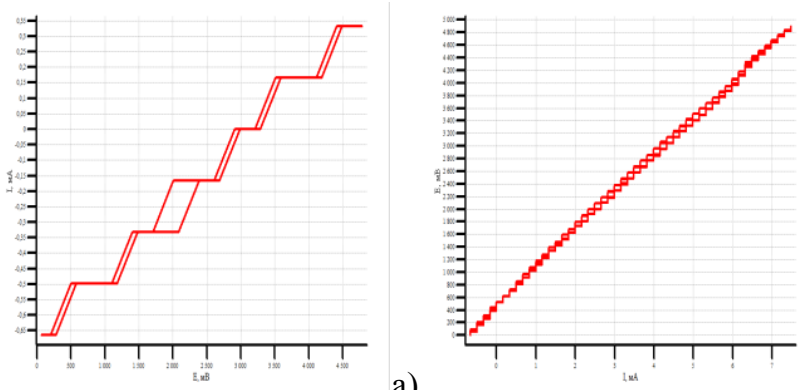

a)

b)
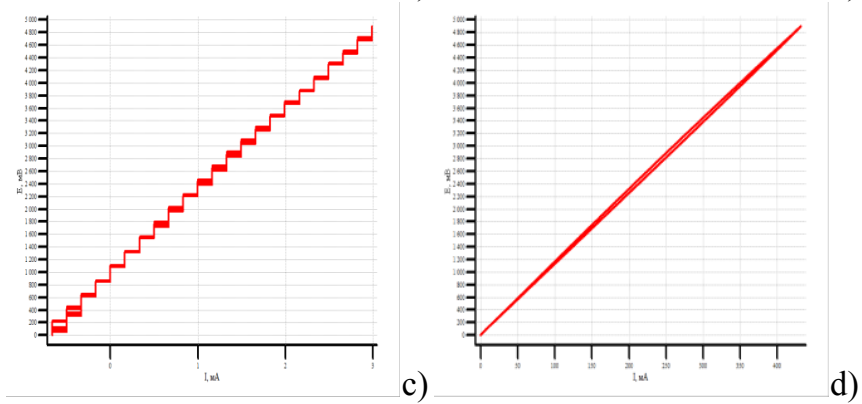

Fig. 6. Current-voltage characteristics ( $U-I$ ) of the a)control samples and with an additive concentration of b) $5 \%$, c) $6 \%$, d) $8 \%$.

\section{Results and Discussion}

During the measurements, the values of the polarization resistance in the samples of cement-sand mortar at the age of 7,21 days were obtained. An experimental linear dependence of the potential on the current strength is obtained (Fig.6) in the specified range of variations for a voltage of $0-5 \mathrm{~V}$ and a current of up to $3 \mathrm{~A}$. Experimentally confirmed that the resistance-concentration relationship is percolation. The concentration of the additive was determined to reach the percolation threshold. 


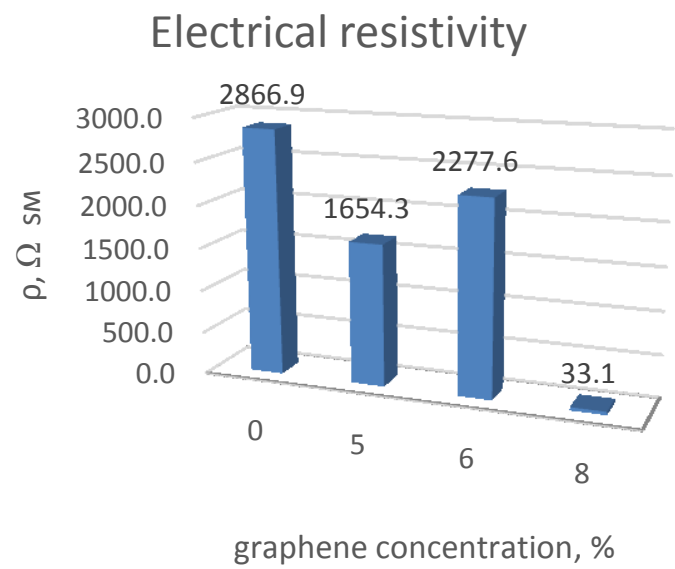

Fig. 7. Resistivity of samples aged 7 days.

\section{Electrical resistivity}

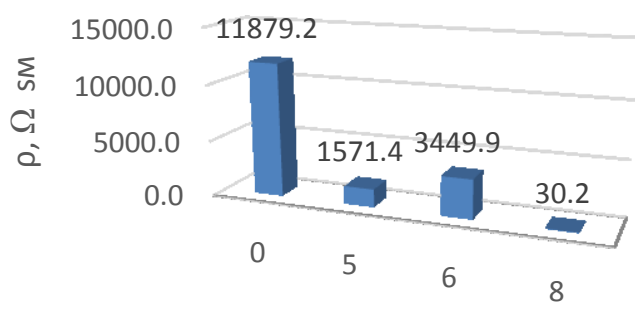

graphene concentration, \%

Fig. 8. Resistivity of samples aged 21 days.

\section{Conclusions}

The electrical resistivity of samples with a graphene concentration of $8 \%$ has a rather low value. The low graphene content ensures the preservation of the strength characteristics of the solution. The obtained conductivity parameters are sufficient for use in compositions for the manufacture of heating elements for structures whose icing entails a violation of operational characteristics. Taking into account the fact that graphene is produced in Belarus, as well as its low cost, all this makes it possible to use this material in the construction and reconstruction of buildings and structures for various purposes.

\section{References}

1. I.Y. Denisyuk, M.V. Uspenskaya, M.I. Fokina, K.Y. Logushkova Electrically conductive concrete composition//Scientific and Technical Bulletin of Information Technologies, Mechanics and Optics. 2018. vol. 18. No. 1. pp. 158-162 doi: 10.17586/2226-1494-2018$18-1-158-162$ 
2. Yoo D.-Y., You I., Lee S.-J. Electrical properties of cementbased compo-sites with carbon nanotubes, graphene, and graphite nanofibers // Sensors. 2017. V. 17. N 5. P. 1064. doi: 10.3390/s17051064

3. Banthia, N.; Djeridane, S.; Pigeon, M. Electrical resistivity of carbon and steel microfiber reinforced cements. Cem. Concr. Res. 1992, 22, 804-814.

4. Loamrat, K.; Sappakittipakorn, M.; Sukontasukkul, P.; Banthia, N. Effect of carbon fiber and graphite powder on resistivity of cement-based sensor under compression. KMUTNB Int. J. Appl. Sci. Technol. 2014, 7, 29-35.

5. Kim, G.M.; Yang, B.J.; Cho, K.J.; Kim, E.M.; Lee, H.K. Influences of CNT dispersion and pore characteristics on the electrical performance of cementitious composites. Compos. Struct. 2017, 164, 32-42.

6. Chebotin V. N. \& Perfilyev M. V. Elektrokhimiya tverdykh elektrolitov [Solid-State Electrochemistry], ed. V. N. Chebotin. Moscow, Khimiya, 1978. 312 p.

7. Sheykin A.Ye. \& Dobshits L. M. Tsementnyy beton vysokoy morozostoykosti [Highly Frost-Resistant Cement Concrete]. Leningrad, Stroyizdat, 1989.128 p.

8. Vrublevsky L.E. // Possibilities of using electrically conductive concrete in civil construction, The review was prepared by the Zonal Research Institute of Standard and Experimental Design of Residential and Public buildings (SIBZNIEP). onethousandninehundredseventyone

9. Vasilyuk Yu. I.; Surkov A.V.; Ivanova T. A. Analysis of modern technologies for the construction of anti-bridging roads using electrically conductive concrete. "MODERN TRENDS IN EDUCATION AND CONSTRUCTION" VI scientific and methodological conference on October 28, 2020. 